\title{
A simple and efficient CRISPR/Cas9 platform for induction of single and multiple, heritable mutations in barley (Hordeum vulgare L.)
}

\author{
Sebastian Gasparis ${ }^{1 *} \mathbb{0}$, Maciej Kała' ${ }^{1}$, Mateusz Przyborowski ${ }^{1}$, Leszek A. Łyżnik², Wacław Orczyk ${ }^{3}$ \\ and Anna Nadolska-Orczyk ${ }^{1}$
}

\begin{abstract}
Background: Genome editing of monocot plants can be accomplished by using the components of the CRISPR/ Cas9 (clustered regularly interspaced short palindromic repeat/CRISPR associated Cas9) technology specifically optimized for these types of plants. Here, we present the development of RNA-guided Cas 9 system for simplex and multiplex genome editing in barley.

Results: We developed a set of customizable RNA-guided Cas9 binary vectors and sgRNA modules for simplex and multiplex editing in barley. To facilitate the design of RNA-guided Cas9 constructs, the pBract derived binary vectors were adapted to Gateway cloning and only one restriction enzyme was required for construction of the sgRNA. We designed a synthetic, codon optimized Cas9 gene containing the N terminal SV40 nuclear localization signal and the UBQ10 Arabidopsis 1st intron. Two different sgRNAs were constructed for simplex editing and one polycistronic tRNA-gRNA construct (PTG) for multiplex editing using an endogenous tRNA processing system. The RNA-guided Cas 9 constructs were validated in transgenic barley plants produced by Agrobacterium-mediated transformation. The highest mutation rate was observed in simplex editing of the cytokinin oxidase/dehydrogenase HvCKX1 gene, where mutations at the hvck 1 locus were detected in $88 \%$ of the screened $T_{0}$ plants. We also proved the efficacy of the PTG construct in the multiplex editing of two CKX genes by obtaining 9 plants (21\% of all edited plants) with mutations induced in both $H_{v C K X}$ and $H_{v} C K X X$. Analysis of the $T_{1}$ lines revealed that mutations in the HvCKX1 gene were transmitted to the next generation of plants. Among 220 screened $T_{1}$ plants we identified 85 heterozygous and 28 homozygous mutants, most of them bearing frameshift mutations in the HvCKX1 gene. We also observed independent segregation of mutations and the Cas9-sgRNA T-DNA insert in several T, plants. Moreover, the knockout mutations of the Nud gene generated phenotype mutants with naked grains, and the phenotypic changes were identifiable in $\mathrm{T}_{0}$ plants.
\end{abstract}

Conclusions: We demonstrated the effectiveness of an optimized RNA-guided Cas9 system that can be used for generating homozygous knockout mutants in the progeny of transgenic barely plants. This is also the first report of successful multiplex editing in barley using a tRNA processing system.

Keywords: CRISPR/Cas9, Genome editing, Barley, PTG, CKX genes

\footnotetext{
*Correspondence: s.gasparis@ihar.edu.pl

${ }^{1}$ Department of Functional Genomics, Plant Breeding and Acclimatization Institute - National Research Institute, 05-870 Radzików, Błonie, Poland

Full list of author information is available at the end of the article
} 


\section{Introduction}

The progress in DNA sequencing technology with increasing speed, scalability at relatively low cost combined with advanced bioinformatics led to accumulation of immense amount of data on genomic sequences, annotated genes and transcriptome profiles of large number of diverse species. The knowledge of plant genomes opens new possibilities for both genetic studies and crop improvement by genetic engineering techniques. However, this knowledge cannot be fully utilized without appropriate research tools for fast and accurate structural/functional analysis and annotation of the sequenced genes. The utilization of various reverse genetics concepts had been limited until the emergence of a breakthrough genome editing technology based on the application of programmable sequence specific endonucleases. In practice, an endonuclease can be modified to acquire specific DNA binding domains and then transferred to the nucleus where it generates double strand DNA breaks (DSB) in a site-specific manner (reviewed by Puchta and Fauser [1]). Until 2013, the most popular genome editing procedures utilized zinc finger nucleases (ZFN) [2] and transcription activator-like effector nucleases (TALENs) [3, 4]. ZFNs and TALENs have not been widely adopted due to the complexities of their design and synthesis. The emergence of the CRISPR/Cas9 (clustered regularly interspaced short palindromic repeat/ Cas9-associated) system has revolutionized genome editing technologies. Simplicity, efficiency, and versatility of this system makes it a popular genetic tool. It was adapted from the prokaryotic adaptive immunity systems, which act against invading foreign DNA [5]. Unlike FokI-based site specific nucleases, Cas9 endonucleases are monomeric proteins with two nuclease domains that cut DNA 3 bp upstream of the PAM motif (Protospacer Adjacent Motif). The specificity is conferred by a $20 \mathrm{nt}$ fragment of gRNA (guide RNA), which binds to the complementary target sequence (protospacer). The presence of a double strand break generated by Cas9 induces intracellular mechanisms of DNA repair, which can be divided into two major groups: homology-directed repair (HDR) and non-homologous end joining (NHEJ). In the HDR mechanism, a broken DNA molecule is repaired via homologous recombination; therefore, the template DNA is required to restore the original sequence or to introduce a specific mutation. In the NHEJ mechanism the free ends of DNA are joined via non-homologous (illegitimate) recombination. As a result of errors in the DSB repair process, small insertions, deletions, or rearrangements may occur at the joining site. If the DSB is generated inside the open reading frame, the error-prone DSB repair may lead to knockout mutations. In somatic cells the NHEJ is a predominant repair mechanism, as opposed to meiotic cells, where DSBs are repaired by homologous recombination [6]. The sequences of Cas9 and sgRNA (single guide RNA) are the only elements of the CRISPR/Cas9 system, which need to be expressed in host cells They can be introduced on a single T-DNA construct. Moreover, the CRISPR/Cas9 system can be used for multiplex editing of different target sequences, as multiple sgRNAs can be transcribed from a single polycistronic gene [7].

The CRISPR/Cas9 system was initially tested in model plants such as Arabidopsis, tobacco, and rice [8-14]. Thereafter, the studies were extended on the application of CRISPR/Cas9 to crop species. Since then, various RNA-guided Cas9 platforms have been developed for more efficient editing and to facilitate the technical aspects of the construction of custom RNA-guided Cas9 vectors (reviewed in [15-17]). In cereals, the studies involving the RNA-guided Cas9 system were focused mainly on three of the most important species: rice $[7,11,13,18-24]$, maize $[25-28]$ and wheat [29-32].

With its sequenced diploid genome, barley may be considered as a diploid model species for closely related hexaploid cereals. There are two reports of RNA-guided Cas9 based genome editing in this crop plant. Lawrenson et al. [33] investigated the target specificity of the RNA-guided Cas9 system in barley by targeting two copies of the HvPM19 gene and observed induced mutations in $23 \%$ and $10 \%$ of the $\mathrm{T}_{0}$ lines, respectively. The mutations were stably transmitted to $\mathrm{T}_{2}$ plants independently of the T-DNA construct. Moreover, the authors reported off-target mutations in non-target copies of HvPM19. Kapusi et al. [34] used five different sgRNA constructs for targeting the ENGase gene in barley by both biolistic and Agrobacterium-mediated transformation. Mutations in the ENGase gene were detected in 25 out of 32 primary transformants in seven lines produced by particle bombardment and 18 lines derived from Agrobacterium-transformation.

For our studies, we choose $H \nu C K X 1$ and $H \nu C K X 3$, two genes belonging to a small family of genes encoding cytokinin oxidase/dehydrogenase enzymes that regulate endogenous levels of cytokinin hormones $[35,36]$. The CKX genes show diversified developmental and tissue specific expression patterns [37, 38], suggesting their specialized functions in diverse plant tissues. The $C K X 1$ and CKX3 knock-out lines may serve as interesting objects for further studies. The function of the ethylene response transcription factor gene Nud is known (it controls the formation of hulled grains [39]), and therefore, we chose it to validate the efficacy of our RNA-guided Cas9 constructs in generating phenotypic changes in barley mutant plants. 
Here, we present analternative version of the RNAguided Cas9 system designed for genome editing in barley. A relatively high rate of induced mutations was achieved by using a synthetic, codon optimized, and intron-enhanced Cas9 gene in combination with an efficient Agrobacterium-transformation method. The design of the binary vectors was also optimized to minimize the cloning steps. Only one restriction enzyme BsaI was required for construction of the sgRNA cassette, which was subsequently integrated into the Cas9 binary vector by Gateway cloning. Using our RNA-guided Cas9 constructs we were able to produce $\mathrm{T}_{0}$ plants with visible phenotypic changes and homozygous, transgene-free mutants could be selected in the $T_{1}$ generation. This is also the first report of multiplex editing in barley using an endogenous tRNA processing system.

\section{Methods}

\section{Design of the Cas9 gene for expression in barely cells}

The sequence of the Cas9 gene from Streptococcus pyogenes (Gene Bank accession AMH03982) was optimized for expression in monocot plants using the GeneOptimizer program (Thermo Fisher Scientific). The GC content was increased from 35.1 to $56.5 \%$ and the amino acid codon usage was adjusted to that observed in maize (https://www.kazusa.or.jp/codon). In order to facilitate translocation of the Cas9 endonuclease protein from cytoplasm to nucleus, a version of the SV40 (simian virus 40) large $\mathrm{T}$ antigen NLS (nuclear localization signal) (NLS-SV40, MAPKKKRKVG) sequence was added to the N-terminus of the Cas9 protein. The Arabidopsis' 310 nt UBQ10 1st intron was placed at +196 within an AGGT canonical splice site sequence of the Cas9 coding sequence. The UBQ10 1st intron is among the best studied enhancing introns in plants [40]. The enhancing effect of this intron in conjunction with the maize ubi promoter was tested in barely plants, while integrated at the +165 position in the luciferase gene used for testing the enhancement effect [41]. An additional restriction site-NcoI- was also incorporated into the $5^{\prime}$ flanking sequence together with the consensus Kozak's sequence (CCGCCATGG) around the ATG start codon. Three internal SacI and one HindIII restriction sites were mutagenized, while preserving the amino acid sequence of the Cas9 protein.

The complete nucleotide sequence of Cas9 is shown in the Additional file 1: Methods S1. Construction of the RNA-guided Cas9 vector.

For cloning Cas9 into the pBract211 vector, the SphI restriction site was removed and two unique restriction sites for the XmaI and SpeI enzymes were added to both sides of the Cas 9 coding sequence. The resulting $4464 \mathrm{bp}$ Cas9 sequence was synthesized and cloned into the
XmaI/SpeI restriction site of the pBract211 vector [42]. The pBract211 vector containing the Cas9 gene was then converted to a Gateway compatible destination vector (pBract211-Cas-GW) using the Gateway conversion kit (Invitrogen). The vector was cut at the SphI site and the Gateway cassette was cloned directly after the nos terminator. This site is used for Gateway cloning of a sgRNA cassette with a chosen target sequence. The detailed cloning protocol of the RNA-guided Cas9 vector is described in Additional file 1: Methods S1.

\section{Design of the sgRNA}

The sequences of the wheat U6 promoter (GenBank accession X63066.1) and gRNA scaffold [13] were synthesized as a single sequence containing a short insert between them with two BsaI restriction sites in the opposite orientation (see Additional file 1: Methods S1 for the complete sequence). To facilitate the detection of induced mutations each target sequence contained the restriction site overlapping the cutting site generated by Cas $93 \mathrm{bp}$ upstream of the PAM motif. The target sequence in the form of two annealed oligos was cloned between the U6 promoter and gRNA scaffold at the BsaI cleavage site. The target sequence of the annealed oligos was forward $5^{\prime}-\mathrm{CTTG}(\mathrm{N})_{20}-3^{\prime}$ and reverse $5^{\prime}$-AAAC $(\mathrm{N})_{20}-3^{\prime}$ (Fig. 1, Additional file 1: Methods S1). The detailed protocol of oligo annealing and sgRNA ligation, and the target sequences used in this study are described in the Additional file 1: Methods S1 and Table S2. The sgRNA cassette was then cloned into the Gateway entry vector. We used the pCR8/GW/TOPO vector, however any Gateway compatible entry vector with attL1/attL2 sites could be used.

\section{Design of the PTG cassette}

A polycistronic tRNA-gRNA cassette was designed for a simultaneous targeting of the $H \nu C K X 1$ and $H \nu C K X 3$ genes using a single U6-gRNA construct. The sgRNA elements were flanked by glycine-tRNA sequences from barley (ASM32608v1:7:24838229:24838299:1) and cloned between the TaU6 promoter and gRNA scaffold after cutting it with the $B s a$ I restriction enzyme. The sequences of TaU6 promoter and gRNA scaffold were the same as for sgRNA. The sequence of the PTG construct is shown in Table S2 and the detailed assembly protocol is described in Additional file 1: Methods S1.

\section{Agrobacterium-mediated transformation}

Immature embryos of barley cv Golden Promise were transformed using the protocol of Harwood et al. [43] with modifications. The donor plants were grown in a controlled environmental chamber at $18{ }^{\circ} \mathrm{C}$ day and $12{ }^{\circ} \mathrm{C}$ night temperatures, and a $16 \mathrm{~h}$ photoperiod with 

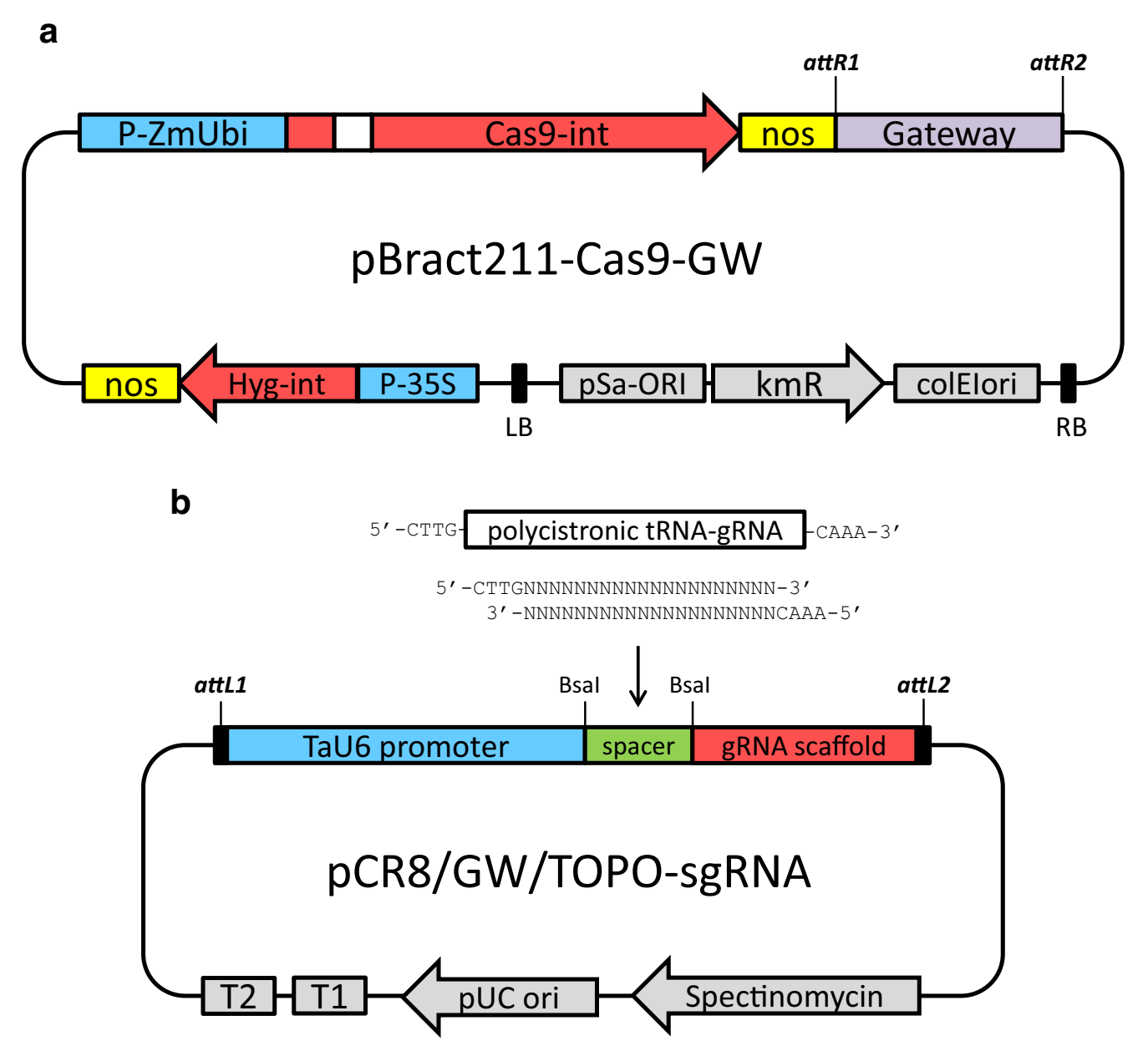

C

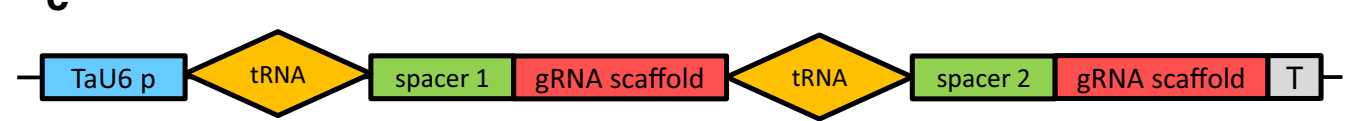

Fig. 1 Schematic description of RNA-guided Cas9 constructs designed for genome editing. a Structure of the binary vector plasmid based on pBract211 used to deliver Cas9:sgRNA components into barley plants. The Gateway cloning site is replaced by the sgRNA cassette; P-ZmUbi, maize ubiquitin promoter; Cas9-int, synthetic gene of nuclease Cas9 with an intron and nuclear localization signal; nos, nopaline synthase terminator; P-35S, CaMV35S promoter; Hyg-int, hygromycin resistance gene; kmR, kanamycin resistance gene. b Structure of the pCR8/GW/TOPO-sgRNA vector used for assembling the sgRNA or PTG constructs. Either oligo duplex for simplex editing or polycistronic tRNA-gRNA (PTG) for multiplex editing can be cloned between the U6 promoter and gRNA scaffold using Bsal-generated overhangs. c Structure of the polycistronic tRNA-gRNA unit with two spacers used for multiplex editing

$350 \mu \mathrm{mol} / \mathrm{m}^{2} / \mathrm{s}$ light intensity provided by fluorescent lamps. The same conditions were applied to regenerated transgenic $T_{0}$ plants. The immature embryos were isolated from surface-sterilized caryopses, and after excising the embryonic axes were placed on callus induction medium (CI) (see Additional file1: Table S3 for medium composition) scutellum side up. On the same day the embryos were inoculated with Agrobacterium tumefaciens culture with the addition of $200 \mu \mathrm{M}$ of acetosyringone. The Agrobacterium culture was grown overnight on MGL medium without antibiotics at $28{ }^{\circ} \mathrm{C}$. Directly after inoculation the embryos were transferred to fresh CI medium, scutellum side down, and co-cultivated for 3 days in the dark. After co-cultivation the embryos were transferred to fresh CI plates containing hygromycin as a selective agent and Timentin to eliminate Agrobacterium from the culture. The embryos and emerging calli were cultured at $22-23^{\circ} \mathrm{C}$ in the dark for 6 weeks, and passed to fresh selection CI medium every 2 weeks. Embryoderived calli were transferred to the transition medium 
(TR) and incubated at $22-23^{\circ} \mathrm{C}$ for $2-3$ weeks under low light conditions that were achieved by covering the plates with two sheets of filter paper. Small regenerating shoots, which emerged during this time, were transferred to regeneration medium (Reg) and cultured under full light conditions until they formed $2-3 \mathrm{~cm}$ plantlets with small roots. The plantlets were next transferred from plates to glass jars with half-strength MS medium without growth regulators but still containing hygromycin and Timentin. Plants, which formed a strong root system, were planted into soil. For the first 2 weeks the plants in the pots were covered with transparent glass jars for acclimation to new conditions. The putative transgenics were PCR screened for the presence of a T-DNA fragment using primers specific to the hpt gene (see Additional file 1: Table S1).

\section{Detection of mutations induced by RNA-guided Cas 9}

For each PCR-positive transgenic plant leaf tissue samples were collected for extraction of genomic DNA. Tissue fragments were collected from possibly every leaf to minimize the risk of false negatives in case of chimeric plants. Genomic DNA was extracted using a standard CTAB method [44]. The target genes were amplified with specific primers flanking the designed target sequence (see Additional file 1: Table S1 for primer sequences) using Q5 Hot-start polymerase (NEB). After amplification, $10 \mu \mathrm{l}$ of the PCR mixture was taken for restriction digestion with the BsmAI ( $H v C K X 1$ and $N u d$ ) or BanII ( $H v C K X 3)$ enzymes. The digested amplicons were separated on $1.5 \%$ agarose gels and imaged on a Kodak Gel Logic 200 Imaging System. PCR products from samples with mutations detected on the gel were cloned into the pGEM-T Easy vector (Promega) for sequencing. At least 10 clones from each sample were sequenced.

\section{Statistical analysis}

The confidence level for segregation ratios of T-DNA in $\mathrm{T}_{1}$ lines was calculated in Excel spreadsheet using Chi squared test.

\section{Results}

Strategy for simplex and multiplex genome editing of barley based on an optimized Cas9 and gRNA module.

An optimized synthetic Cas9 gene based on the native sequence from Streptococcus pyogenes was used for mutagenesis experiments in barely cells. The optimization process included the adjustment of the coding sequence GC content and amino acid codon usage according to that observed in monocot plants. In addition to other minor modifications, the $310 \mathrm{bp}$ UBQ10-i1 intron from Arabidopsis thaliana was placed within the $5^{\prime}$ coding region of Cas9. The recombined Cas9-encoding gene was cloned to the pBract211-derived binary vector for plant genetic transformation and the resulting construct was designated as pBract211-Cas9 (Fig. 1a).

To introduce sgRNA, the second component of the RNA-guided Cas9 system, the pBract211-Cas9 vector was converted into the Gateway destination vector.

The Gateway cassette containing the attR1 and attR2 recombination sites was placed directly after the nos terminator within the T-DNA fragment (Fig. 1a, Additional file 1: Methods S1).

Construction of this intermediate vector facilitated the next cloning step, in which the complete sgRNA cassette consisting of the U6 or U3 promoter, spacer, gRNA scaffold, and U6 or U3 terminator was introduced into the vector by Gateway $L R$ reaction.

We decided to use this method for two reasons: (1) to avoid restriction cloning of both the sgRNA and spacers directly into the pBract 211 vector with incompatible restriction enzymes and (2) to facilitate the production of multiple sets of pBract211-Cas9 vectors with different variants of sgRNA cassettes (e.g. for simplex or multiplex editing, or with different RNA promoters). This ensures more versatility of our system, as one can use the existing gRNA constructs, which are shared in repositories or exchanged between laboratories.

The sgRNA module for simplex editing was designed similar to the strategy described by Shan et al. [13]. The DNA fragment consisting of the U6 RNA promoter from wheat and the sgRNA sequence with transcription termination signal was synthesized and cloned into the pCR8/ GW/TOPO intermediate vector. The final pCR8/GW/ TOPO Gateway entry vector was obtained by inserting a $20 \mathrm{bp}$ spacer complementary to the target sequence between the U6 promoter and gRNA scaffold. The spacer was inserted as an annealed oligo using type-II restriction endonucleases which produces minimal 4 nt overhangs (Fig. 1b, Fig. S2).

We decided to use $B s a \mathrm{I}$ instead of $B b s \mathrm{I}$, as this enzyme is compatible with Golden Gate cloning vectors. The RNA transcripts driven by the U6 promoter start with G, therefore the optimal target sequence should conform to the pattern $\mathrm{G}(\mathrm{N})_{19} \mathrm{NGG}$, however, it is not obligatory as the $(\mathrm{N})_{20} \mathrm{NGG}$ spacers also work.

For multiplex editing we decided to use the tRNA processing system-based strategy proposed by Xie et al. [7]. This mechanism can be used to simultaneously produce multiple gRNAs from a single polycistronic tRNA-gRNA gene (PTG). Based on this strategy we assembled a PTG gene with two different gRNAs fused with two glycine-tRNA sequences from barley (ASM 32608v1:7:24838229:24838299:1) (Fig. 1c, Additional file 1: Table S2). Both sgRNA cassettes and the PTG gene were cloned into the pBract211-Cas9 binary vector. In summary, we prepared a set of three RNA-guided Cas9 
transformation vectors, two of them for simplex editing and one with the PTG cassette for multiplex editing. As targets we chose three barley genes, $H \nu C K X 1, H \nu C K X 3$, and $\mathrm{Nud}$. Mutated $\mathrm{Nud}$ alleles (nud) produce naked (hulles) grains with non-adhering hulls, thus the $\mathrm{Nud}$ gene was targeted to generate mutants with naked grains.

Validation of the RNA-guided Cas9 vectors for simplex and multiplex editing in transgenic barley plants.

To test the efficiency of our RNA-guided Cas9 constructs in generating targeted mutations at a single genomic site (locus), we produced transgenic barley plants after Agrobacterium-transformation with two vectors, pBract211-Cas9-ckx1 and pBract211-Cas9nud with gRNAs targeting the $H v C K X 1$ and $N u d$ genes, respectively. The target sequences were chosen near the $5^{\prime}$ end of the ORF to increase the chance of reading frame disruption caused by induced mutations, and we selected those where the cutting site of Cas9 was overlapped by a restriction site. After transformation, all independent transgenic $T_{0}$ plants were screened by PCR amplification and restriction analysis (PCR/RE) of the amplified fragments for mutations at the target sequences. Since most indels generated by the NHEJ repair system should disrupt the restriction site overlapping the double-strand break site, the mutation is confirmed by the presence of an uncut band on an agarose gel, while the wild-type copies of the gene are cut by the enzyme (Fig. 2). Using this approach, we detected 47 (66\%) transgenic plants with mutations in the $H v C K X 1$ gene and 18 (64\%) with mutations in the Nud gene (Fig. 2a, b, Table 1).

The disadvantage of restriction analysis is its potential inaccuracy, as some indels could be generated upstream or downstream of the diagnostic restriction site, which may lead to false negative bands on the gel. Therefore, to validate the results of the $\mathrm{PCR} /$ restriction analysis, we cloned and sequenced the amplicons from 25 randomly selected $\mathrm{T}_{0}$ plants transformed with the ckx1-sgRNA cassette. Ten clones per plant were sequenced. The mutations were detected in 22 out of 25 plants, which gave $88 \%$ efficiency (number of $\mathrm{T}_{0}$ plants testing positive for the mutation events). Moreover, the sequencing confirmed the presence of single nucleotide insertions or deletions, which did not disrupt the BsmAI restriction enzyme site used for the initial PCR/restriction a

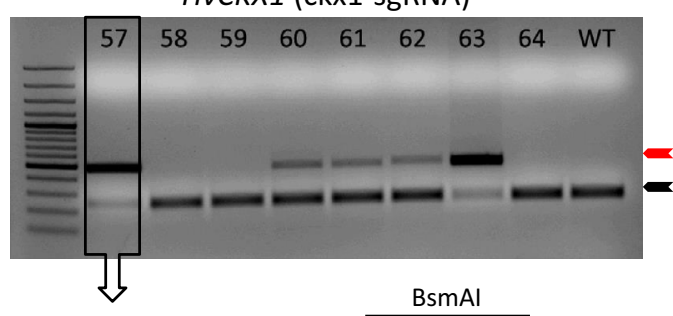

HVCKX1 GTCGCTCTGATCACCGCGGCGTCTC-CTACGGCGCACG

\#1 GTCGCTCTGATCACCGCGGCGTCTCACTACGGCGCACG 1 bp ins

\#2 GTCGCTCTGATCACCGCGGCGT----CTACGGCGCACG 3 bp del

b
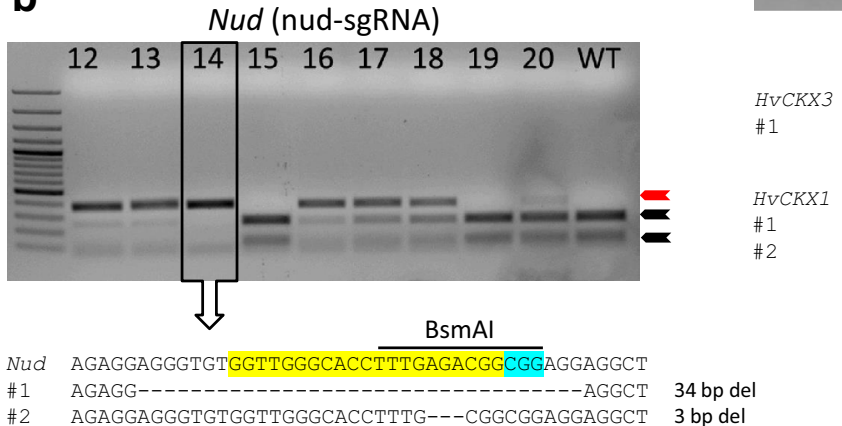

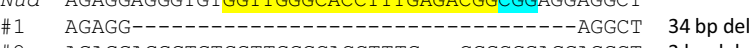

\#2 AGAGGAGGGTGTGGTTGGGCACCTTTG---CGGCGGAGGAGGCT 3 bp del

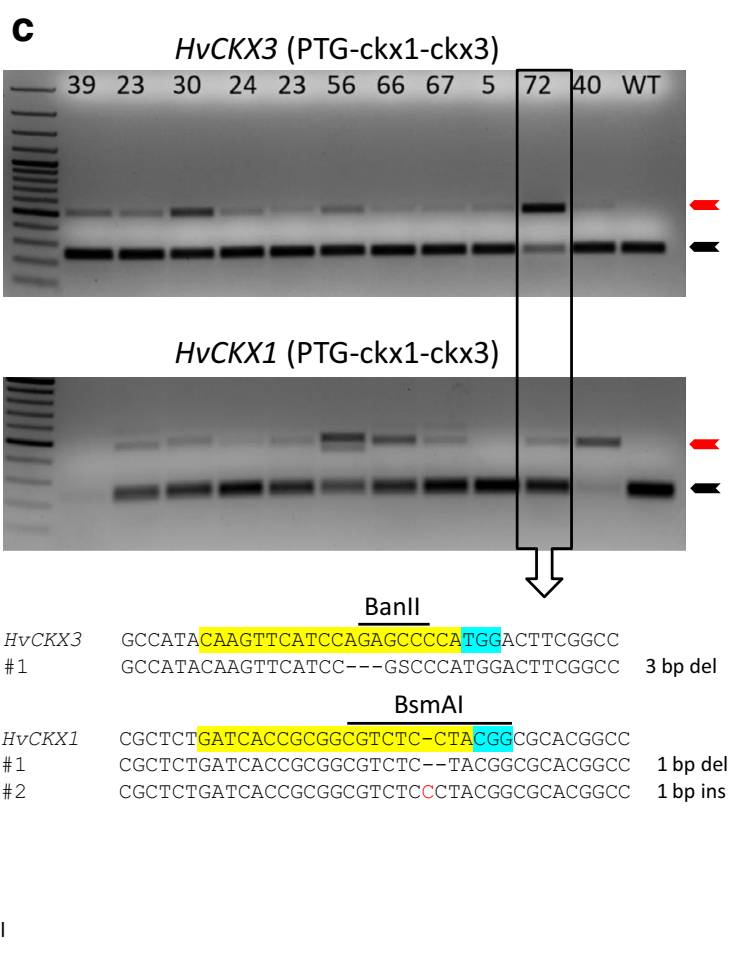

Fig. 2 PCR/RE screening of $T_{0}$ plants transformed with Cas9:sgRNA constructs for simplex editing of HvCKX1 (a), Nud (b), and the PTG construct for multiplex editing of the HVCKX1 and HvCKX3 genes (c). PCR products of the appropriate target gene were treated by restriction enzymes that overlap the potential mutation site (indicated above the aligned sequences). Red arrowheads indicate uncut bands of amplicons with mutations; black arrowheads indicate bands of wild-type sequences cut by an enzyme. PCR products from selected samples were cloned and sequenced to identify the pattern of mutations; target sequences for sgRNA:Cas9 are marked in yellow, PAM motifs are marked in light blue; deletions are indicated by dashes and insertions by red letters 
Table 1 Summary of genetic transformation of barley with different sgRNA constructs and PCR/RE screening of transformants for the presence of induced mutations

\begin{tabular}{lllllc}
\hline sgRNA construct & Edited gene & No. of explants & $\begin{array}{l}\text { No. of transgenic } \\
\text { events (\%) }\end{array}$ & $\begin{array}{l}\text { No. of independent } \\
\text { transgenic plants }\end{array}$ & $\begin{array}{l}\text { No. of PCR-RE } \\
\text { detected } \\
\text { mutants }\end{array}$ \\
\hline ckX1-sgRNA & HvCKX1 & 1143 & $137(12)$ & 71 & 47 \\
nud-sgRNA & Nud & 1209 & $96(8)$ & 28 & 18 \\
PTG-ckX1-ckX3 & HvCKX1 & 1320 & $142(11)$ & 72 & 49 \\
& HvCKX3 & & & & $9^{a}$ \\
\hline
\end{tabular}

${ }^{a}$ No. of plants with mutations in both the $H v C K X 1$ and $H v C K X 3$ genes

analysis (Additional file 2: Fig. S3). Interestingly, most of the tested plants appeared to be chimeric for the introduced mutations (more than two different mutations found in one $\mathrm{T}_{0}$ sample). Taking aside a sampling error, the $\mathrm{T}_{0}$ plants \#34, \#54, and \#70 could represent the biallelic heterozygous events (only two different mutations found), while the $\mathrm{T}_{0}$ plant \#63 could indicate the biallelic homozygous event (all mutations the same) (Additional file 2: Fig. S3). To further estimate the status of the $T_{0}$ mutant plants, we checked the ratio between the mutated and wild-type copies of the $H \nu C K X 1$ gene in the $\mathrm{T}_{0}$ mutants. The wild-type copies of the $H \nu C K X 1$ gene were found in 5 out of 22 mutated plants (Table 2). The most frequent type of induced mutations was a deletion or

Table 2 Distribution of Cas9:sgRNA induced mutations in the HvCKX1 gene in $\mathrm{T}_{0}$ plants and their segregation in $\mathrm{T}_{1}$ progeny

\begin{tabular}{|c|c|c|c|c|c|c|c|}
\hline \multirow[t]{2}{*}{$T_{0}$ plant ID } & \multicolumn{2}{|c|}{$\begin{array}{l}\text { No. of mutant and wt }{ }^{\mathrm{a}} \text { clones } \\
\text { in a } \mathrm{T}_{0} \text { plant }^{\mathrm{b}}\end{array}$} & \multicolumn{3}{|c|}{$\begin{array}{l}\text { Segregation of mutations in } \mathrm{T}_{1} \text { lines } \\
\text { [No. of wt, ht, and hm plants] }\end{array}$} & \multirow{2}{*}{$\begin{array}{l}\text { Segregation of T-DNA } \\
\text { in } \mathrm{T}_{1} \text { lines } \\
\mathrm{PCR}(+): P C R(-)\end{array}$} & \multirow[t]{2}{*}{$\begin{array}{l}\text { No. of mutated, T-DNA } \\
\text { free plants in } T_{1} \text { lines }\end{array}$} \\
\hline & Mutant & $w t^{b}$ & wt & ht & $\mathrm{hm}$ & & \\
\hline 22 & 10 & 0 & 0 & 5 & 5 & $7: 3$ & 3 \\
\hline 27 & 10 & 0 & 7 & 2 & 1 & $2: 8$ & 3 \\
\hline 28 & 10 & 0 & 10 & 0 & 0 & $8: 2$ & 0 \\
\hline 29 & 9 & 1 & 3 & 5 & 2 & $7: 3$ & 3 \\
\hline 30 & 10 & 0 & 7 & 3 & 0 & $8: 2$ & 0 \\
\hline 32 & 10 & 0 & 6 & 4 & 0 & $8: 2$ & 1 \\
\hline 33 & 10 & 0 & 8 & 2 & 0 & $3: 7$ & 0 \\
\hline 34 & 10 & 0 & 0 & 1 & 9 & $10: 0$ & 0 \\
\hline 37 & 8 & 2 & 4 & 6 & 0 & $4: 6$ & 2 \\
\hline 38 & 10 & 0 & 0 & 8 & 2 & $9: 1$ & 1 \\
\hline 41 & 9 & 1 & 6 & 4 & 0 & $5: 5$ & 2 \\
\hline 42 & 10 & 0 & 5 & 5 & 0 & $7: 3$ & 0 \\
\hline 44 & 8 & 2 & 1 & 8 & 1 & $9: 1$ & 0 \\
\hline 45 & 10 & 0 & 6 & 4 & 0 & $9: 1$ & 0 \\
\hline 47 & 7 & 3 & 6 & 4 & 0 & $9: 1$ & 0 \\
\hline 54 & 10 & 0 & 6 & 3 & 1 & $6: 4$ & 1 \\
\hline 57 & 10 & 0 & 2 & 5 & 3 & $7: 3$ & 2 \\
\hline 63 & 10 & 0 & 5 & 3 & 2 & $7: 3$ & 1 \\
\hline 67 & 10 & 0 & 6 & 4 & 0 & $8: 2$ & 1 \\
\hline 70 & 10 & 0 & 7 & 3 & 0 & $7: 3$ & 0 \\
\hline 78 & 10 & 0 & 6 & 2 & 2 & $8: 2$ & 0 \\
\hline 80 & 10 & 0 & 6 & 4 & 0 & $8: 2$ & 0 \\
\hline \multicolumn{3}{|c|}{ Total number of $\mathrm{T}_{1}$ plants: } & 107 & 85 & 28 & $156: 64$ & 20 \\
\hline
\end{tabular}

a wt wild-type, $h$ t heterozygous, $h m$ homozygous

b Mutations detected in leaf tissue 
insertion of a single nucleotide. However, larger deletions were observed in seven plants, some of them exceeding $20 \mathrm{bp}$, and the largest was the deletion of a $58 \mathrm{bp}$ fragment (Additional file 2: Fig. S3).

To validate the functionality of the RNA-guided Cas9 construct for multiplex editing, we designed the PTG gene with two gRNAs targeting the HvCKX1 and $H \nu C K X 3$ genes, respectively (Fig. 1c). The construct in the pBract211-Cas-PTG vector was used to generate transgenic barley plants by Agrobacterium-mediated transformation. As previously, $\mathrm{T}_{0}$ transformants were first screened by PCR and restriction analysis and then sequenced (Fig. 2c). The mutation frequency in the $H \nu C K X 1$ gene was comparable to that generated by a single sgRNA $49(68 \%)$, but it was considerably lower in $H \nu C K X 313$ (18\%) (Table 1). Nevertheless, the overall efficiency of the PTG construct was still satisfactory, as we obtained 9 plants (21\% of all mutated plants) with mutations in both CKX genes.

\section{Inheritance and segregation of induced mutations in the $T_{1}$ generation}

The inheritance and segregation of $H v C K X 1$ mutations was analyzed in the progeny of the 22 mutant self-pollinated $\mathrm{T}_{0}$ plants. Ten $\mathrm{T}_{1}$ progeny plants, from each $\mathrm{T}_{0}$ mutant plant, were screened for the presence of mutations. No mutations were found only in the progeny of the $\mathrm{T}_{0}$ plant \#28 (Table 2). Identical mutations (long deletions) transmitted from $T_{0}$ to $T_{1}$ plants were found in three $\mathrm{T}_{0} / \mathrm{T}_{1}$ lines (i.e. \#22, \#34, and \#78) (Fig. 3). Among $113 \mathrm{~T}_{1}$ mutants, eighty five were heterozygous (only one allele mutagenized), while the remaining 28 were homozygous/biallelic plants (found within $10 \mathrm{~T}_{1}$ lines), which gave approx. 3 homozygous plants per line. Among these 10 lines we found $26 \mathrm{~T}_{1}$ homozygous plants with frameshift mutations (see Additional file 3: Fig. S4). The predominant, non-Mendelian pattern of inheritance and the presence of wild-type alleles in the $T_{1}$ generation, even from $\mathrm{T}_{0}$ plants originally identified as biallelic (\#34, $\# 54, \# 70$, \#63), indicated the chimeric status of $\mathrm{T}_{0}$ transgenic plants (Table 2). We also tested to what extent the induced mutations and T-DNA from the RNA-guided Cas9 vector segregated independently in the $T_{1}$ generation. This allowed for discrimination of $T_{1}$ individuals with inherited primary mutations from those in which the mutation could be potentially created de novo, because of the expression of Cas9 and sgRNA. 17.7\% (20 out of 113) of $\mathrm{T}_{1} \mathrm{~T}$-DNA-free individuals inherited the primary mutation in $H v C K X 1$ gene. T-DNA segregation in the $\mathrm{T}_{1}$ generations followed the Mendelian 3:1 ratio (at confidence level $\mathrm{P}=0.99, \alpha=0.05$ ) in 12 out of 22 original $\mathrm{T}_{0}$ events indicating a single-locus insertion of T-DNA in those plants (Table 2). The segregation ratio 9:1 or 10:0 could indicate not linked integration of two copies of T-DNA in the additional $5 \mathrm{~T}_{0}$ plants. 2 out of 20 T-DNA free mutants were homozygotes in terms of the mutation in the $H \nu C K X 1$ gene. This proved that it was possible to select non-transgenic mutants in the $T_{1}$ generation by screening at least 10 plants from each $\mathrm{T}_{1}$ line.

\section{Generation of mutants with phenotypic changes}

Typical domesticated barley cultivars have covered (hulled) grains with the hull firmly adhering to the caryopsis at maturity. However, there are a few cultivars, which produce naked caryopses also known as naked barley. This trait is controlled by a single locus $(n u d)$ on chromosome 7HL. We used a sgRNA construct targeting the Nud gene in the Golden Promise hulled cultivar to generate putative mutants with naked caryopses. The mutations were induced in 18 out of 25 transgenic $\mathrm{T}_{0}$ plants. The phenotypic changes in the form of naked caryopses with no adhering hull were observed in 11 mutants (Fig. 4a, b) which indicates for the presence of biallelic mutations in these plants. To check the mutations pattern, fragment of the $N u d$ gene was sequenced in seven selected mutants. The frameshift mutations

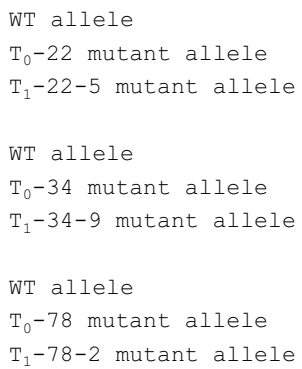

TGGTCGCTCTGATCACCGCGGCGTCTCCTACGGCGCACGGCCAGACGTGGCACGGCGA

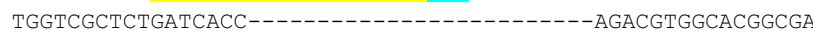

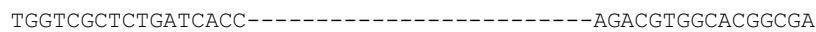

TCGTTCACGTGTTACTGGTCGCTCTGATCACCGCGGCGTCTCCTACGGCGCACGGCCA

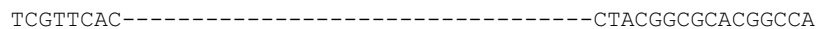
TCGTTCAC-------------

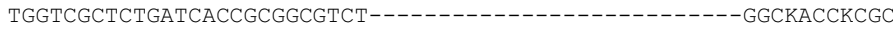

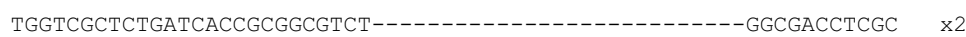

Fig. 3 Transmission of Cas9:sgRNA induced mutations from $T_{0}$ to $T_{1}$ lines. Sequence alignment of wild-type $H v C K X 1$ and the mutant alleles identified within the same germline. The numbers of homozygous $T_{1}$ plants with the same mutant allele are indicated on the right; the target sequence is marked in yellow and PAM motif in light blue; deletions are indicated by dashes 
a

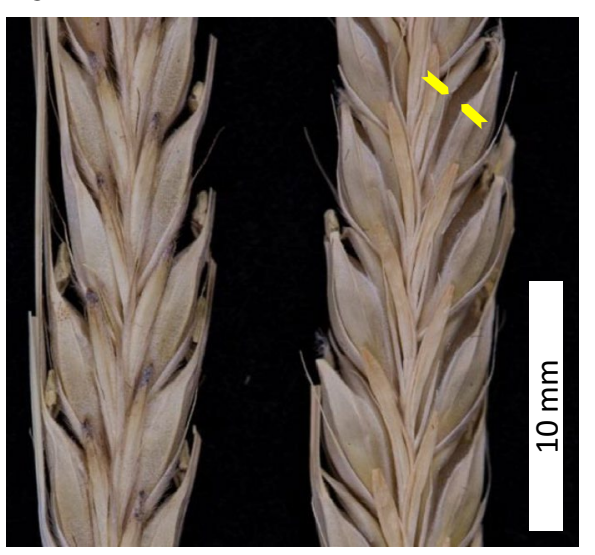

b

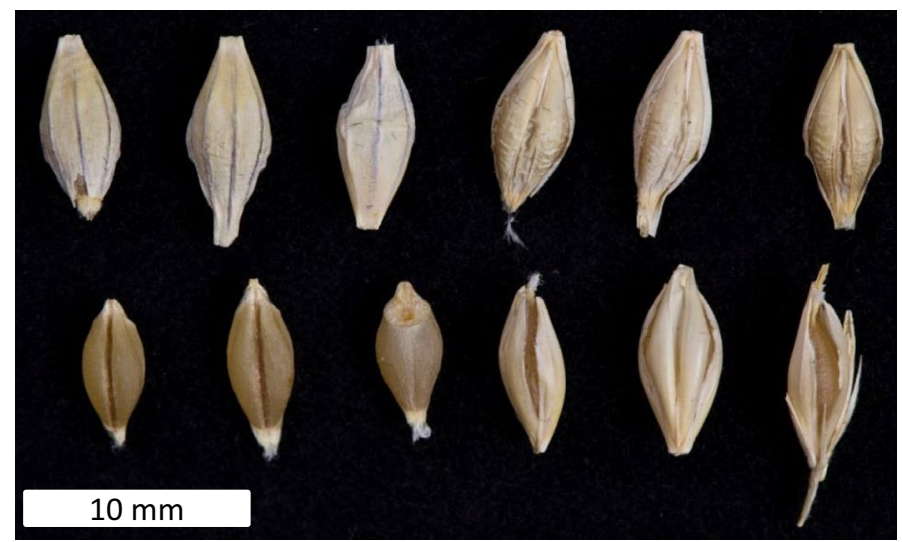

C

WT

$14-1$

TACAGGAAGAGGAGGGTGTGGTTGGGCACCTTTGAGACGGCGGAGGAGGCTGCGCG

TWCMGGARGAGG------------------------------AGGCTGCGCG $\times 2$

TACAGGAAGAGGAGGGTGTGGTTGGGCACCTTTG---CGGCGGAGGAGGCTGCGCG х2

34 bp del

3 bp del

WT TACAGGAAGAGGAGGGTGTGGTTGGGCACCTTTGAGA-CGGCGGAGGAGGCTGCGCG

43-1 TACAGGAAGAGGAGGGTGTGGTTGGGCACCTTTGAGATCGGCGGAGGAGGCTGCGCG $\times 5$

1 bp ins

WT TACAGGAAGAGGAGGGTGTGTTGGGCACCTTTGAGA-CGGCGGAGGAGGCTGCGCG

85-1 TACAGGAAGAGGAGGGTGTGGTTGGGCACCTTTGAGACCGGCGGAGGAGGCTGCGCG $\times 4$

85-2 TACAGGAAGAGGAGGGTGTGGTTGGGCACCTTTGAGAACGGCGGAGGAGGCTGCGCG $\mathrm{x} 1$

1 bp ins

1 bp ins

Fig. 4 Phenotype changes generated by knock-out mutations of the Nud gene in barley. a Mature spikes of wild-type control (left) and nud mutant (right) plants; yellow arrowheads indicate opened hulls surrounding a naked caryopsis (all grains in the spike are naked but the hull openings are visible only at a specific angle). b Examples of mature covered grains from a wild-type plant (upper row) and naked grains from a nud mutant plant (bottom row); c sequence alignment of the Nud gene fragments cloned from $\mathrm{T}_{0}$ biallelic mutants. Target sequence is marked in yellow and PAM motif in light blue; deletions are indicated by dashes, and insertions by red letters

were detected in all seven plants. Moreover, two plants, \#14 and \#85 possessed heterozygous biallelic mutations (two different mutations), and homozygous biallelic mutation (all mutations the same) was detected only in plant \#43 (Fig. 4c). Interestingly, the wild-type amplicons were detected in the remaining four mutants, which indicates for the chimeric status of these plants (see Additional file 4: Fig. S5).

\section{Discussion}

Genome editing has become a powerful tool for functional genomics studies in plants because it allows for the precise introduction of mutations at specific genomic locations. With its outstanding efficiency, reliability, and simplicity, the RNA-guided Cas9 system quickly became the most popular technique among other available genome editing methods, however its efficiency depends on the plant species. A large number of RNAguided Cas9 edited Arabidopsis thaliana plants with a mutation frequency up to $90 \%$ can be easily produced by Agrobacterium-infiltration of floral buds [10, 45]. In contrast, the first attempts of RNA-guided Cas9-based editing of some monocot species resulted in significantly lower mutation frequencies, such as $8.2 \%$ in rice [13], $5.6 \%$ in wheat [31], and up to $23 \%$ in barley [33]. These results indicated that the RNA-guided Cas9 system required some optimization to achieve greater mutation rates in monocots. One factor to consider is the use of appropriate binary vectors in combination with efficient protocols for stable Agrobacterium-mediated transformation. In this report, the pBract-based customizable binary vectors and gRNA modules were used. As targets we chose the $C K X$ genes encoding the cytokinin oxidase/ dehydrogenase enzymes, which permanently inactivate endogenous cytokinins. Taking into account the significance of this physiological process, we chose two $C K X$ genes, $H \nu C K X 1$ and $H \nu C K X 3$, differing in tissue specific expression patterns, of which potential knockout effects may be further studied. In contrast, the ethylene response transcription factor gene Nud was chosen to estimate 
the efficiency of the RNA-guided Cas9 system to produce visible phenotypical changes in barley, as this gene controls whether the barley grains are covered (hulled) or naked (hulles). Most of the barley cultivars including Golden Promise produce hulled grains with hulls strictly adhering to caryopses. In turn, some varieties with mutated Nud alleles ( $n u d$ ) produce naked (hulles) grains with non-adhering hulls. Taketa et al. [39] proved that the naked caryopsis phenotype is caused by either natural or induced mutations in the ethylene response (ERF) family transcription factor gene $\mathrm{Nud}$. Thus, the $\mathrm{Nud}$ gene was targeted to generate mutants with naked grains.

Expression of the Cas9 gene, the use of monocot specific promoters, and an efficient transformation protocol are other factors that may affect the efficiency of the RNA-guided Cas9 system in generating knock-out, heritable mutations in barely. Strong expression of endonuclease Cas9 is desirable in order to generate a large number of double strand breaks at specific genomic locations. This can be achieved by plant codon optimization of the native Cas9 sequence in combination with monocot specific promoters. The positive relationship between codon usage and translation efficiency of heterologous proteins in plants has been established leading to, for example, 100-fold increase of crylA protein accumulation in transgenic tobacco and tomato [46, 47]. Codon optimization has become a standard practice for heterologous proteins needed to be expressed at high levels in plants, e.g. plant-made pharmaceuticals (reviewed by $[48,49])$. Some researchers indicated that use of plant codon optimized Cas9 under monocot specific promoters, such as the maize $U b i$ promoter resulted in greater mutant efficiency, which ranged from 60 to $100 \%$ in rice $[7,20]$, maize [26, 27], and barley (this study), as compared to the range of 8.6-45.3\%, if human codon optimized Cas 9 and/or the $35 \mathrm{~S}$ promoter were used [18, 19, 33]. The expression of heterologous genes in transgenic plants can also be affected by using genetic regulatory elements such as introns. Intron mediated enhancement (IME) of gene expression in plants is a well-known phenomenon (reviewed by Laxa [50]). We used the UBQ10i1 intron from Arabidopsis, which is among the best studied enhancing introns in plants [40]. These types of promoter-proximal introns increase the expression levels of transgenes in both dicot and monocot plants if they are positioned at the $5^{\prime}$ coding region [51]. The enhancing effect of the UBQ10-i1 intron has been proven in barley when used in the luciferase gene along with the Ubi1 promoter [41]. The optimal expression of sgRNA, the second component of the RNA-guided Cas9 system, may also be important. In plants, polymerase III promoters such as U3 and U6 are frequently used for the expression of sgRNAs. In our system, the U6 promoter from wheat, which efficacy was proven in several studies [13, 31-33], has been used to drive expression of sgRNA

Our RNA-guided Cas9 system was developed based on the pBract 211 vector. The pBract vectors are improved successors of the pGreen vector family, which have been widely used for transformation of cereals. The advantage of $\mathrm{pBract}$ vectors is their optimization for monocot plant transformation protocols. They exploit a maize ubiquitin promoter for stable and constitutive transgene expression, and contain the hpt gene for hygromycin selection of transformants. We have used both the pGreen and pBract vectors for transformation of even weakly susceptible cultivars of oat, wheat, and triticale [52-54]. An efficient genetic transformation method is required to produce an adequate number of edited plants with desirable mutations. For cereals, the Agrobacterium-mediated transformation seems to be more effective and reliable method as compared to the particle bombardment procedures [55-57]. The transformation efficiency ranging from 8 to 12\% is routine for Agrobacterium-transformation of the barely Golden Promise cultivar in our laboratories [43]. The mutation frequency in simplex editing of the $H v C K X 1$ gene reached up to $88 \%$, which is similar to the efficiency accomplished in model plants such as Arabidopsis [26, 45] and rice [21], and was sufficient to select homozygous mutants with frameshift mutations in the $\mathrm{T}_{1}$ generation. The previously reported Cas9-based mutation rates in barley obtained after Agrobacteriummediated transformation ranged from $23 \%\left(23 \mathrm{~T}_{0}\right.$ plants analyzed) [33] to $78 \%\left(20 \mathrm{~T}_{0}\right.$ plants analyzed) [34]. Moreover, the number of biallelic mutations induced in the Nud gene allowed for the selection of $\mathrm{T}_{0}$ transformants with identifiable knockout phenotypes.

It is worth to acknowledge that also other platforms have been utilized for genome editing of barley. Meganuclease I-SceI was successfully used for gene replacement by homology-directed repair [58]. TALEN technology was validated in barley by targeting phytase promoter [59] and $g f p$ transgene [60, 61]. Holme et al. [62] evaluated the mature grain phytase candidate using both TALEN and RNA-guided Cas9 systems. Interestingly, the similar mutation rate of $43-44 \%$ was reported for both platforms and the induced mutations were stably inherited to the $\mathrm{T}_{2}$ generation. The ZFN and TALEN platforms have also been used, to a minor extent, in other important cereal species, such as rice [63-65], maize [25, 66] and wheat [31]. Although the performance of these platforms is relatively low as compared to the CRSIPR/Cas9 system, they still can be useful for specific purposes.

Simultaneous editing of multiple target sequences is desired for a more complex gene editing experimentation such as deletion of large chromosomal fragments. It is also particularly useful for knockout of multiple 
homologous or homeologous genes in polyploid species. As opposed to ZFN and TALENs, the RNA-guided Cas9 system is more flexible and it can be utilized for multiplex editing, since the Cas9 and sgRNAs are transcribed separately and multiple gRNA modules can be put into a single T-DNA construct. Recently, a polycistronic tRNA-gRNA system has been developed for multiplex RNA-guided Cas9-based editing in plants, which relies on endogenous mechanisms of tRNA processing [7]. In eukaryotic cells, tRNA precursors are cleaved at specific sites by RNase $\mathrm{P}$ and RNase $\mathrm{Z}$ to remove excess $5^{\prime}$ and 3 'sequences. It has been suggested that this mechanism is used to produce tRNAs and other small RNAs such as small nucleolar RNA (snoRNA) from a single polycistronic gene. Similarly, multiple gRNAs can be assembled together with flanking tRNA sequences into a single polycistronic tRNA-gRNA gene (PTG) which requires only one U3 or U6 promoter. This greatly simplifies the RNA-guided Cas 9 construct design by minimizing the sequential cloning steps needed for separate gRNAs. The effectiveness of PTG constructs in multiplex genome editing have been demonstrated in rice [7] and maize [28], where it has been proven that PTG-based system was more efficient in mutagenesis than single sgRNAs. In maize, the editing rate of tRNA-gRNA units was from 85.7 to $100 \%$ as compared to $57-71 \%$ of U6-sgRNAs in simplex editing [28]. In rice, the editing rate of tRNAgRNA units was from 47 to $100 \%$ as compared to $44-60 \%$ of U3-sgRNAs. Moreover, up to $76 \%$ of mutations generated by tRNA-gRNA units were biallelic, whereas the U3-sgRNAs generated only $20 \%$ [7]. The similar positive effect of increased editing rate was observed in two yeast organisms, i.e. O. polymorpha [67] and Y. lipolytica [68] where the tRNA-based transcript processing was used together with Cas9 for genome editing. Another advantage of the PTG system is the fact that target sequences used in tRNA-gRNA units may start with any nucleotide, whereas sgRNA transcripts of U3 and U6 promoters are obligated to start with A and G nucleotide respectively. In this study, we designed a PTG construct for simultaneous editing of two different $C K X$ genes using a tRNA sequence from barley. Our results indicate that PTGbased multiplex editing can be successfully used in barley, however, the achieved editing rate ranging from 18 to $68 \%$ was slightly lower than in rice and maize (from 47 to $100 \%)[7,28]$. Even though the mutation frequency in the $C K X 3$ gene was markedly reduced, the overall efficiency of the PTG construct was still sufficient to produce plants with mutations in both $C K X$ genes.

The differences in the mutation rates between the CKX1 (68\%) and CKX3 (18\%) genes are of interest, since they are not produced by the different arrangement of the PTG construct. Presumably, they are affected by the properties of the target sequences. It has been demonstrated that the GC content of the target sequences has significant impact on the sgRNA cleavage efficiency [19, 69-72]. Therefore, the greater efficiency of $C K X 1 \mathrm{sgRNA}$ may result from its greater GC content (65\%) as compared to CKX3 sgRNA (55\%). Moreover, both genes are located at distinct loci and show different tissue-dependent expression patterns. The highest expression level of $H \nu C K X 1$ was observed in developing grains [38], whereas $H v C K X 3$ in roots (data not shown). It could be possible that the highly transcribed $H \nu C K X 1$ locus is more exposed to the sgRNA/Cas9 complex as opposed to the transcriptionally inactive $H \nu C K X 3$ locus. All these factors should be taken into consideration when designing sgRNAs.

The analysis of $\mathrm{T}_{1}$ plants revealed that RNA-guided Cas9-induced mutations were transmitted to the next generation in at least $50 \%$ of $\mathrm{T}_{1}$ lines. However, only in three lines the segregation of inherited mutation coincided with Mendelian rules. The non-Mendelian segregation observed in other lines can be explained by the chimeric nature of $\mathrm{T}_{0}$ plants. Our results indicated that different types of mutations can be induced independently in somatic and generative tissues in $\mathrm{T}_{0}$ plants. Since the mutations in the $\mathrm{T}_{0}$ plants were detected in leaf tissue, there is no information whether these mutations were also induced in generative tissues and transmitted to gametes. This fact may explain an observation showing that wild-type $\mathrm{T}_{1}$ plants are a progeny of $\mathrm{T}_{0}$ mutants, where no wild-type clones of the $H \nu C K X 1$ gene were detected in the sampled leaf tissues (Table 2). Therefore, it is impossible to predict the segregation of mutations in $\mathrm{T}_{1}$ progeny based only on the analysis of somatic tissue. Based on T-DNA segregation analysis we found at least one plant with a mutation inherited independently from the Cas9-sgRNA T-DNA in $50 \%$ of $\mathrm{T}_{1}$ lines. The segregation of non-transgenic mutants is very important in view of commercial applications of genome editing technology in the countries where such plants are excluded from the GMO legislation.

\section{Conclusions}

The ability to create genetic mutants is essential for the study of gene function in plants. In recent years, efforts have been made to adapt the bacterial CRISPR/Cas9 system for its application to crop plants. In this report we demonstrated the effectiveness of an optimized RNAguided Cas9 system in the genome editing of barley. The developed set of binary vectors offers a simple, inexpensive, time-saving, and efficient way to create genome edited plants with single or multiple heritable mutations. The performed optimizations and universality of the system allows for its application to other cereal species. 


\section{Additional files}

Additional file 1: Methods S1. Figure S1. Schematic view of the pBract211 binary vector. Figure S2. Cloning site for the pCR8/GW/TOPOsgRNA vector. Table S1. List of PCR primers and oligonucleotides used in this study. Table S2. List of target sequences used for genome editing in barley. Table S3. Composition of media for Agrobacterium-mediated transformation and in vitro regeneration of barley immature embryos.

Additional file 2: Figure S3. Sequence alignment of the HVCKX1 gene fragments cloned from selected $T_{0}$ plants. Target sequence is marked in yellow and PAM motif in light blue; deletions are indicated by dashes.

Additional file 3: Figure S4. Sequence alignment of the HvCKX1 gene fragments cloned from selected $T_{1}$ plants. Target sequence is marked in yellow and PAM motif in light blue; deletions are indicated by dashes.

Additional file 4: Figure S5. Sequence alignment of the Nud gene fragments cloned from selected $T_{0}$ plants. Target sequence is marked in yellow and PAM motif in light blue; deletions are indicated by dashes.

\section{Authors' contributions}

SG designed the experiments. LA $\downarrow$ designed the Cas9 gene. SG, MK, MP performed the experiments. SG, LAŁ, WO, AN-O analyzed the data and wrote the manuscript. All authors read and approved the final manuscript.

\section{Author details}

${ }^{1}$ Department of Functional Genomics, Plant Breeding and Acclimatization Institute - National Research Institute, 05-870 Radzików, Błonie, Poland

2 Department of Plant Genetics, Breeding and Biotechnology, Warsaw University of Life Sciences (SGGW), 02-776 Warsaw, Poland. ${ }^{3}$ Department of Genetic Engineering, Plant Breeding and Acclimatization Institute - National Research Institute, 05-870 Radzików, Błonie, Poland.

\section{Acknowledgements}

This work was supported by the National Research Centre of Poland, grant no. UMO-2015/17/D/NZ9/02020.

\section{Competing interests}

The authors declare that they have no competing interests.

\section{Availability of data and materials}

All data generated or analyzed during this study are included in this published article (and its supplementary information files).

\section{Consent for publication}

Not applicable.

Ethics approval and consent to participate

Not applicable.

\section{Funding}

This research was financed by the National Research Centre of Poland, Grant No. UMO-2015/17/D/NZ9/02020.

\section{Publisher's Note}

Springer Nature remains neutral with regard to jurisdictional claims in published maps and institutional affiliations.

Received: 26 April 2018 Accepted: 10 December 2018

Published online: 18 December 2018

\section{References}

1. Puchta $H$, Fauser $F$. Synthetic nucleases for genome engineering in plants: prospects for a bright future. Plant J. 2014;78(5):727-41.

2. Lloyd A, Plaisier CL, Carroll D, Drews GN. Targeted mutagenesis using zinc-finger nucleases in Arabidopsis. Proc Natl Acad Sci USA. 2005;102(6):2232-7.
3. Boch J, Scholze H, Schornack S, Landgraf A, Hahn S, Kay S, Lahaye T, Nickstadt A, Bonas U. Breaking the code of DNA binding specificity of TAL-type III effectors. Science. 2009;326(5959):1509-12.

4. Cermak T, Doyle EL, Christian M, Wang L, Zhang Y, Schmidt C, Baller JA, Somia NV, Bogdanove AJ, Voytas DF. Efficient design and assembly of custom TALEN and other TAL effector-based constructs for DNA targeting. Nucleic Acids Res. 2011;39(12):e82.

5. Jinek M, Chylinski K, Fonfara I, Hauer M, Doudna JA, Charpentier E. A programmable dual-RNA-guided DNA endonuclease in adaptive bacterial immunity. Science. 2012;337(6096):816-21.

6. Puchta $\mathrm{H}$. The repair of double-strand breaks in plants: mechanisms and consequences for genome evolution. J Exp Bot. 2005;56(409):1-14.

7. Xie K, Minkenberg B, Yang Y. Boosting CRISPR/Cas9 multiplex editing capability with the endogenous tRNA-processing system. Proc Natl Acad Sci USA. 2015;112(11):3570-5.

8. Feng Z, Zhang B, Ding W, Liu X, Yang DL, Wei P, Cao F, Zhu S, Zhang F, Mao $Y$, et al. Efficient genome editing in plants using a CRISPR/Cas system. Cell Res. 2013;23(10):1229-32.

9. Li JF, Norville JE, Aach J, McCormack M, Zhang D, Bush J, Church GM, Sheen J. Multiplex and homologous recombination-mediated genome editing in Arabidopsis and Nicotiana benthamiana using guide RNA and Cas9. Nat Biotechnol. 2013;31(8):688-91.

10. Mao Y, Zhang H, Xu N, Zhang B, Gou F, Zhu JK. Application of the CRISPR-Cas system for efficient genome engineering in plants. Mol Plant. 2013;6(6):2008-11.

11. Miao J, Guo D, Zhang J, Huang Q, Qin G, Zhang X, Wan J, Gu H, Qu LJ. Targeted mutagenesis in rice using CRISPR-Cas system. Cell Res. 2013;23(10):1233-6.

12. Nekrasov V, Staskawicz B, Weigel D, Jones JD, Kamoun S. Targeted mutagenesis in the model plant Nicotiana benthamiana using Cas9 RNAguided endonuclease. Nat Biotechnol. 2013;31(8):691-3.

13. Shan Q, Wang Y, Li J, Zhang Y, Chen K, Liang Z, Zhang K, Liu J, Xi JJ, Qiu JL, et al. Targeted genome modification of crop plants using a CRISPR-Cas system. Nat Biotechnol. 2013;31(8):686-8.

14. Xie K, Yang Y. RNA-guided genome editing in plants using a CRISPR-Cas system. Mol Plant. 2013;6(6):1975-83.

15. Ding YD, Li H, Chen LL, Xie KB. Recent advances in genome editing using CRISPR/Cas9. Front Plant Sci. 2016;7:703.

16. Khatodia S, Bhatotia K, Passricha N, Khurana SM, Tuteja N. The CRISPR/Cas genome-editing tool: application in improvement of crops. Front Plant Sci. 2016;7:506

17. Ma XL, Zhu QL, Chen YL, Liu YG. CRISPR/Cas9 platforms for genome editing in plants: developments and applications. Mol Plant. 2016;9(7):961-74

18. Xu R, Li H, Qin R, Wang L, Li L, Wei P, Yang J. Gene targeting using the Agrobacterium tumefaciens-mediated CRISPR-Cas system in rice. Rice (N Y). 2014;7(1):5.

19. Zhang H, Zhang J, Wei P, Zhang B, Gou F, Feng Z, Mao Y, Yang L, Zhang $\mathrm{H}, \mathrm{Xu}$ N, et al. The CRISPR/Cas9 system produces specific and homozygous targeted gene editing in rice in one generation. Plant Biotechnol J. 2014;12(6):797-807.

20. Zhou H, Liu B, Weeks DP, Spalding MH, Yang B. Large chromosomal deletions and heritable small genetic changes induced by CRISPR/Cas9 in rice. Nucleic Acids Res. 2014;42(17):10903-14.

21. Ma X, Zhang Q, Zhu Q, Liu W, Chen Y, Qiu R, Wang B, Yang Z, Li H, Lin $Y$, et al. A robust CRISPR/Cas9 system for convenient, high-efficiency multiplex genome editing in monocot and dicot plants. Mol Plant. 2015;8(8):1274-84.

22. Endo M, Mikami M, Toki S. Biallelic gene targeting in rice. Plant Physiol. 2016:170(2):667-77

23. Sun Y, Zhang X, Wu C, He Y, Ma Y, Hou H, Guo X, Du W, Zhao Y, Xia L. Engineering herbicide-resistant rice plants through CRISPR/Cas9-mediated homologous recombination of acetolactate synthase. Mol Plant. 2016:9(4):628-31.

24. Xu RF, Yang YC, Qin RY, Li H, Qiu CH, Li L, Wei PC, Yang JB. Rapid improvement of grain weight via highly efficient CRISPR/Cas9-mediated multiplex genome editing in rice. J Genet Genom. 2016;43(8):529-32.

25. Liang Z, Zhang K, Chen K, Gao C. Targeted mutagenesis in Zea mays using TALENs and the CRISPR/Cas system. J Genet Genom. 2014;41(2):63-8. 
26. Xing HL, Dong L, Wang ZP, Zhang HY, Han CY, Liu B, Wang XC, Chen QJ. A CRISPR/Cas9 toolkit for multiplex genome editing in plants. BMC Plant Biol. 2014;14:327.

27. Svitashev S, Young JK, Schwartz C, Gao H, Falco SC, Cigan AM. Targeted mutagenesis, precise gene editing, and site-specific gene insertion in maize using Cas9 and guide RNA. Plant Physiol. 2015;169(2):931-45.

28. Qi WW, Zhu T, Tian ZR, Li CB, Zhang W, Song RT. High-efficiency CRISPR/ Cas9 multiplex gene editing using the glycine tRNA-processing systembased strategy in maize. BMC Biotechnol. 2016;16:58.

29. Upadhyay SK, Kumar J, Alok A, Tuli R. RNA-guided genome editing for target gene mutations in wheat. G3 (Bethesda). 2013;3(12):2233-8.

30. Shan $\mathrm{Q}$, Wang Y, Li J, Gao C. Genome editing in rice and wheat using the CRISPR/Cas system. Nat Protoc. 2014;9(10):2395-410.

31. Wang Y, Cheng X, Shan Q, Zhang Y, Liu J, Gao C, Qiu JL. Simultaneous editing of three homoeoalleles in hexaploid bread wheat confers heritable resistance to powdery mildew. Nat Biotechnol. 2014;32(9):947-51.

32. Gil-Humanes J, Wang Y, Liang Z, Shan Q, Ozuna CV, Sanchez-Leon S, Baltes NJ, Starker C, Barro F, Gao C, et al. High-efficiency gene targeting in hexaploid wheat using DNA replicons and CRISPR/Cas9. Plant J. 2017:89(6):1251-62

33. Lawrenson T, Shorinola O, Stacey N, Li CD, Ostergaard L, Patron N, Uauy C, Harwood W. Induction of targeted, heritable mutations in barley and Brassica oleracea using RNA-guided Cas9 nuclease. Genome Biol. 2015;16:258.

34. Kapusi E, Corcuera-Gómez M, Melnik S, Stoger E. Heritable genomic fragment deletions and small indels in the putative ENGase gene induced by CRISPR/Cas9 in barley. Front Plant Sci. 2017;8:540. https://doi. org/10.3389/fpls.2017.00540.

35. Werner T, Motyka V, Laucou V, Smets R, Van Onckelen H, Schmulling T. Cytokinin-deficient transgenic Arabidopsis plants show multiple developmental alterations indicating opposite functions of cytokinins in the regulation of shoot and root meristem activity. Plant Cell. 2003;15(11):2532-50.

36. Werner T, Schmulling T. Cytokinin action in plant development. Curr Opin Plant Biol. 2009;12(5):527-38.

37. Zalewski W, Galuszka P, Gasparis S, Orczyk W, Nadolska-Orczyk A. Silencing of the HvCKX1 gene decreases the cytokinin oxidase/dehydrogenase level in barley and leads to higher plant productivity. J Exp Bot. 2010;61(6):1839-51.

38. Zalewski W, Gasparis S, Boczkowska M, Rajchel IK, Kala M, Orczyk W, Nadolska-Orczyk A. Expression patterns of HvCKX genes indicate their role in growth and reproductive development of barley. PLOS ONE. 2014;9(12):e115729.

39. Taketa S, Amano S, Tsujino Y, Sato T, Saisho D, Kakeda K, Nomura M, Suzuki T, Matsumoto T, Sato K, et al. Barley grain with adhering hulls is controlled by an ERF family transcription factor gene regulating a lipid biosynthesis pathway. Proc Natl Acad Sci USA. 2008;105(10):4062-7.

40. Rose AB, Elfersi T, Parra G, Korf I. Promoter-proximal introns in Arabidopsis thaliana are enriched in dispersed signals that elevate gene expression. Plant Cell. 2008;20(3):543-51.

41. Bartlett JG, Snape JW, Harwood WA. Intron-mediated enhancement as a method for increasing transgene expression levels in barley. Plant Biotechnol J. 2009;7(9):856-66.

42. BRACT: https://www.jic.ac.uk/technologies/genomic-services/bract/. Accessed 20 Apr 2018

43. Harwood WA, Bartlett JG, Alves SC, Perry M, Smedley MA, Leyl N, Snape JW. Barley transformation using agrobacterium-mediated techniques. In: Jones HD, Shewry PR, editors. Transgenic wheat, barley and oats: production and characterization protocols. Totowa: Humana Press; 2009. p. 137-47.

44. Murray MG, Thompson WF. Rapid isolation of high molecular weight plant DNA. Nucleic Acids Res. 1980;8(19):4321-5.

45. Yan L, Wei S, Wu Y, Hu R, Li H, Yang W, Xie Q. High-efficiency genome editing in arabidopsis using YAO promoter-driven CRISPR/Cas9 system. Mol Plant. 2015;8(12):1820-3.

46. Perlak FJ, Fuchs RL, Dean DA, McPherson SL, Fischhoff DA. Modification of the coding sequence enhances plant expression of insect control protein genes. Proc Natl Acad Sci USA. 1991;88(8):3324-8.

47. Rouwendal GJ, Mendes O, Wolbert EJ, de Douwe de Boer A. Enhanced expression in tobacco of the gene encoding green fluorescent protein by modification of its codon usage. Plant Mol Biol. 1997;33(6):989-99.
48. Desai PN, Shrivastava N, Padh H. Production of heterologous proteins in plants: strategies for optimal expression. Biotechnol Adv. 2010;28(4):427-35

49. Egelkrout E, Rajan V, Howard JA. Overproduction of recombinant proteins in plants. Plant Sci. 2012;184:83-101.

50. Laxa M. Intron-mediated enhancement: a tool for heterologous gene expression in plants? Front Plant Sci. 1977;2016:7

51. Parra G, Bradnam K, Rose AB, Korf I. Comparative and functional analysis of intron-mediated enhancement signals reveals conserved features among plants. Nucleic Acids Res. 2011;39(13):5328-37.

52. Gasparis S, Bregier C, Orczyk W, Nadolska-Orczyk A. Agrobacterium-mediated transformation of oat (Avena sativa L.) cultivars via immature embryo and leaf explants. Plant Cell Rep. 2008;27(11):1721-9.

53. Binka A, Orczyk W, Nadolska-Orczyk A. The Agrobacterium-mediated transformation of common wheat (Triticum aestivum L.) and triticale ( $x$ Triticosecale Wittmack): role of the binary vector system and selection cassettes. Journal of applied genetics. 2012;53(1):1-8.

54. Gasparis S, Kala M, Przyborowski M, OrczykW, Nadolska-Orczyk A. Artificial MicroRNA-based specific gene silencing of grain hardness genes in polyploid cereals appeared to be not stable over transgenic plant generations. Front Plant Sci. 2017;2016:7.

55. Dai SH, Zheng P, Marmey P, Zhang SP, Tian WZ, Chen SY, Beachy RN, Fauquet $C$. Comparative analysis of transgenic rice plants obtained by Agrobacterium-mediated transformation and particle bombardment. Mol Breed. 2001;7(1):25-33.

56. Travella S, Ross SM, Harden J, Everett C, Snape JW, Harwood WA. A comparison of transgenic barley lines produced by particle bombardment and Agrobacterium-mediated techniques. Plant Cell Rep. 2005;23(12):780-9.

57. Zalewski W, Orczyk W, Gasparis S, Nadolska-Orczyk A. HvCKX2 gene silencing by biolistic or Agrobacterium-mediated transformation in barley leads to different phenotypes. BMC Plant Biol. 2012;12:206.

58. Watanabe K, Breier U, Hensel G, Kumlehn J, Schubert I, Reiss B. Stable gene replacement in barley by targeted double-strand break induction. J Exp Bot. 2016:67(5):1433-45.

59. Wendt T, Holm PB, Starker CG, Christian M, Voytas DF, Brinch-Pedersen $\mathrm{H}$, Holme IB. TAL effector nucleases induce mutations at a pre-selected location in the genome of primary barley transformants. Plant Mol Biol. 2013;83(3):279-85.

60. Gurushidze M, Hensel G, Hiekel S, Schedel S, Valkov V, Kumlehn J. Truebreeding targeted gene knock-out in barley using designer TALE-nuclease in haploid cells. PLoS ONE. 2014;9(3):e92046.

61. Budhagatapalli N, Rutten T, Gurushidze M, Kumlehn J, Hensel G. Targeted modification of gene function exploiting homology-directed repair of TALEN-mediated double-strand breaks in barley. G3 (Bethesda). 2015;5(9):1857-63.

62. Holme IB, Wendt T, Gil-Humanes J, Deleuran LC, Starker CG, Voytas DF, Brinch-Pedersen $\mathrm{H}$. Evaluation of the mature grain phytase candidate HvPAPhy_a gene in barley (Hordeum vulgare L.) using CRISPR/Cas9 and TALENs. Plant Mol Biol. 2017;95(1-2):111-21.

63. Cantos C, Francisco P, Trijatmiko KR, Slamet-Loedin I, Chadha-Mohanty PK. Identification of "safe harbor" loci in indica rice genome by harnessing the property of zinc-finger nucleases to induce DNA damage and repair. Front Plant Sci. 2014;5:302.

64. Shan Q, Wang Y, Chen K, Liang Z, Li J, Zhang Y, Zhang K, Liu J, Voytas DF, Zheng $X$, et al. Rapid and efficient gene modification in rice and Brachypodium using TALENs. Mol Plant. 2013;6(4):1365-8.

65. Shan Q, Zhang Y, Chen K, Zhang K, Gao C. Creation of fragrant rice by targeted knockout of the OsBADH2 gene using TALEN technology. Plant Biotechnol J. 2015;13(6):791-800.

66. Char SN, Unger-Wallace E, Frame B, Briggs SA, Main M, Spalding MH, Vollbrecht E, Wang K, Yang B. Heritable site-specific mutagenesis using TALENs in maize. Plant Biotechnol J. 2015;13(7):1002-10.

67. Numamoto M, Maekawa $\mathrm{H}$, Kaneko Y. Efficient genome editing by CRISPR/Cas9 with a tRNA-sgRNA fusion in the methylotrophic yeast Ogataea polymorpha. J Biosci Bioeng. 2017;124(5):487-92.

68. Schwartz CM, Hussain MS, Blenner M, Wheeldon I. Synthetic RNA polymerase III promoters facilitate high-efficiency CRISPR-Cas9-mediated genome editing in Yarrowia lipolytica. ACS Synth Biol. 2016;5(4):356-9.

69. Jiang $W$, Zhou H, Bi H, Fromm M, Yang B, Weeks DP. Demonstration of CRISPR/Cas9/sgRNA-mediated targeted gene modification 
in Arabidopsis, tobacco, sorghum and rice. Nucleic Acids Res. 2013;41(20):e188.

70. Feng Z, Mao Y, Xu N, Zhang B, Wei P, Yang DL, Wang Z, Zhang Z, Zheng R, Yang $L$, et al. Multigeneration analysis reveals the inheritance, specificity, and patterns of CRISPR/Cas-induced gene modifications in Arabidopsis. Proc Natl Acad Sci USA. 2014;111(12):4632-7.
71. Wang T, Wei JJ, Sabatini DM, Lander ES. Genetic screens in human cells using the CRISPR-Cas9 system. Science. 2014;343(6166):80-4.

72. Pan C, Ye L, Qin L, Liu X, He Y, Wang J, Chen L, Lu G. CRISPR/Cas9-mediated efficient and heritable targeted mutagenesis in tomato plants in the first and later generations. Sci Rep. 2016;6:24765.
Ready to submit your research? Choose BMC and benefit from:

- fast, convenient online submission

- thorough peer review by experienced researchers in your field

- rapid publication on acceptance

- support for research data, including large and complex data types

- gold Open Access which fosters wider collaboration and increased citations

- maximum visibility for your research: over $100 \mathrm{M}$ website views per year

At BMC, research is always in progress.

Learn more biomedcentral.com/submissions 